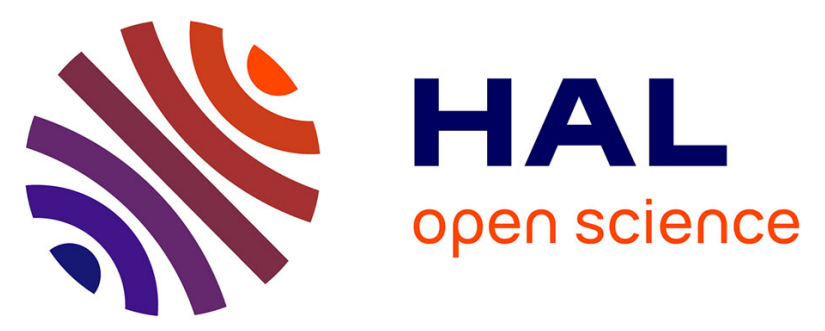

\title{
La place de la physicochimie en urologie et en néphrologie : le bilan de 10ans de collaboration entre physiciens, chimistes et praticiens hospitaliers
}

Dominique Bazin, Emmanuel Letavernier, J.-P. Haymann, P. Méria, Michel Daudon

\section{To cite this version:}

Dominique Bazin, Emmanuel Letavernier, J.-P. Haymann, P. Méria, Michel Daudon. La place de la physicochimie en urologie et en néphrologie : le bilan de 10ans de collaboration entre physiciens, chimistes et praticiens hospitaliers. Progrès en Urologie, 2016, 26 (11-12), pp.608-618. 10.1016/j.purol.2016.08.019 . hal-01371949

\section{HAL Id: hal-01371949 \\ https://hal.sorbonne-universite.fr/hal-01371949}

Submitted on 26 Sep 2016

HAL is a multi-disciplinary open access archive for the deposit and dissemination of scientific research documents, whether they are published or not. The documents may come from teaching and research institutions in France or abroad, or from public or private research centers.
L'archive ouverte pluridisciplinaire HAL, est destinée au dépôt et à la diffusion de documents scientifiques de niveau recherche, publiés ou non, émanant des établissements d'enseignement et de recherche français ou étrangers, des laboratoires publics ou privés. 


\title{
La place de la physicochimie en urologie et en néphrologie : le bilan de 10 ans de collaboration entre physiciens, chimistes et praticiens hospitaliers \\ The role of physicochemistry in urology and nephrology, selected results obtained during the last ten years
}

\author{
D. Bazin ${ }^{\mathrm{a}, *, \mathbf{b}}$, E. Letavernier $^{\mathrm{c}, \mathrm{d}}$, J.-P. Haymann $^{\mathrm{c}, \mathrm{d}}$, P. Méria $^{\mathrm{e}}$, M. Daudon $^{\mathrm{c}, \mathrm{d}}$ \\ ${ }^{a}$ LCMCP, collège de France, Sorbonne universités, UPMC, 11, place Marcelin-Berthelot, 75005 Paris, France \\ ${ }^{\mathrm{b}}$ Laboratoire de physique des solides, Université Paris XI, 91405 Orsay cedex, France \\ c Service d'explorations fonctionnelles, Hopital Tenon, AP-HP, 4, rue de la Chine, 75970 Paris, France \\ dinserm, UMRS 1155, UPMC, Hôpital Tenon, 75970 Paris, France \\ e Service d'urologie, Hôpital St-Louis, 1, avenue C.-Vellefaux, 75010 Paris, France
}

MOTS CLÉS : Physicochimie ; Urologie ; Néphrologie ; Grands instruments ; Rayonnement synchrotron

\section{Résumé}

Introduction. Les calcifications pathologiques rénales qu'elles s'apparentent à des concrétions ou à des calcifications tissulaires impliquent de savoir déterminer correctement les composés chimiques qui les composent afin de porter un diagnostic menant à une prise en charge adéquate. Le but de cet article de revue est de dépeindre les techniques physicochimiques aptes à réaliser cette caractérisation structurale et chimique à l'échelle du micromètre et de montrer leur insertion au sein même de l'hôpital.

Méthode. Les résultats acquis ces dix dernières années utilisant des techniques de caractérisation de laboratoire ou spécifiques aux grands instruments comme le synchrotron Soleil ou le laboratoire LéonBrillouin sont présentés. Leur pertinence dans l'activité du clinicien a été examinée.

Résultats. Le rôle des éléments traces dans la pathogenèse des calculs rénaux au travers de données acquises par fluorescence $\mathrm{X}$ est discuté. Si leurs propriétés catalytiques ne sont pas confirmées par les données expérimentales, l'analyse fine a dévoilé certaines particularités intéressantes concernant les teneurs en $\mathrm{Zn}$ des deux composés d'oxalate de calcium, i.e. whewellite et weddellite. En effet, les calculs de weddellite présentent des bipyramides aux angles et arêtes vifs susceptibles d'induire une inflammation tissulaire. L'analyse morphoconstitutionnelle des biopsies rénales dévoile une grande diversité chimique puisque 22 corps différents ont été identifiés. Cette diversité indique que les méthodes de coloration dont l'anatomopathologiste dispose sont insuffisantes pour caractériser ces corps. Enfin, nous énumérons différentes problématiques abordées par les grands instruments et définies par le clinicien.

Conclusion. La complexité structurale et la diversité chimique des calcifications pathologiques invitent à la mise en œuvre de techniques de caractérisation physicochimiques. Seule cette approche permet au clinicien la pose d'un diagnostic fiable et donc une prise en charge adéquate du patient.

\section{Summary}

Introduction. The presence of pathological calcifications, which can be either concretions or ectopic call for physicochemical characterisation techniques in order to define a significant diagnosis. The aim of this review is to present a set of characterisation techniques able to describe at the micrometer scale their structural and chemical characteristics and show their place at the hospital.

Method. Results already published in the last ten years based on characterisation techniques present in laboratories or specific to large-scale instruments are presented. Their usefulness for the clinician is discussed.

Results. The presence and role of heavy metals in urinary stones through data collected through microX-ray fluorescence is debated. If these data suggest a simple substitution process of calcium, recent data suggest that weddellite is associated to a Zn-rich environment, partly favoured by an inflammation process. Investigation on the chemistry and the structure of unusual deposits in kidney biopsies show a great chemical diversity of ectopic calcifications. Such diversity shows that staining procedures to characterize such deposits are obsolete. Finally, several diseases which can be assessed through techniques specific to large-scale instruments and defined by the clinician are presented.

Conclusion. The chemical and structural complexity of pathological calcifications call for a characterization through physicochemical techniques. Only such approach allows the clinician to define a significant diagnosis and to care the patient. 


\section{Introduction}

Comme le rappelait le Pr. Jacques Friedel [1921-2014], la synergie entre physique, chimie et médecine est au cœur de la recherche de plusieurs scientifiques français de premier plan [1]. Louis Pasteur [1822-1895] combine cristallographie, chimie et optique. Il établit un parallèle entre la morphologie d'un cristal, sa structure cristallographique et la nature de son interaction avec une source de lumière polarisée [2]. Marie Curie [1867-1934], l'une des rares scientifiques à avoir été honorée par deux prix Nobel fonde, à la suite de ses recherches sur la radioactivité, une école de radiologie. Elle forme des jeunes femmes aide-radiologistes et participe activement à la création de 150 postes fixes de radiologie au sein des hôpitaux militaires. Durant le conflit de 1914-1918, elle montera au front pour réaliser des radiographies [3]. Enfin, il convient de rappeler le cours de Charles Friedel [1832-1899] à la Sorbonne qui allia chimie organique et cristallographie [4].

La recherche sur les calcifications pathologiques s'inscrit dans cette veine puisque l'on y conjugue physique, chimie, biologie et médecine. L'observation de leurs singularités structurales et/ou chimiques est à la base d'une activité clinique majeure en néphrologie. Plus précisément, l'analyse morpho-constitutionnelle conçue par M. Daudon permet déposer un diagnostic [5-10]. Dans la mesure où cette caractérisation s'établit à des échelles cellulaires et subcellulaires, cette approche précise les paramètres physicochimiques pertinents inhérents à leur pathogenèse tout en permettant un diagnostic encore plus précoce.

Dans ce manuscrit, nous montrons à partir de nos publications, l'apport de la physicochimie en néphrologie et en urologie.

\section{Méthodes, un aperçu succinct}

\subsection{L'analyse morpho-constitutionnelle, une clé pour l'urologue.}

Cette analyse constitue un fil d'Ariane entre caractérisation routinière à l'hôpital et recherche académique. Pour cette dernière, les expériences de physicochimie sont effectuées entre autre sur des grands instruments comme le centre national de rayonnement synchrotron Soleil (CNRSS) [11] ou le laboratoire de recherche Léon-Brillouin [12]. Le point de départ est toujours lié à une problématique médicale définie par les cliniciens. Cette recherche revient ensuite au niveau du patient en proposant, soit une méthode de diagnostic originale, soit en affinant le traitement médical pour moduler les processus physicochimiques associés aux calcifications pathologiques.

Cette relation entre les caractéristiques physicochimiques d'une calcification et la pathologie qui en est responsable a été définie initialement pour la lithiase. Elle s'esquisse désormais pour d'autres calcifications comme les concrétions prostatiques [13,14], les calcifications ectopiques mammaires [15], pancréatiques [16] ou encore thyroïdiennes [17]. Cette relation transcende ainsi la néphrologie et l'urologie pour coloniser d'autres disciplines médicales en abordant des problèmes de santé publique majeurs.

Cette démarche scientifique s'inscrit dans une nouvelle école dans laquelle les techniques de physicochimie sont présentes au sein même des salles d'opération. Cette proximité au service du clinicien permet d'acquérir un maximum de données biochimiques en temps réel et devrait conduire à une optimisation du geste chirurgical. 


\subsection{Les techniques de physicochimie classiques dans les mains d'un clinicien.}

L'imagerie médicale (scintigraphie, scanner, échographie...) est aujourd'hui incontournable dans de nombreuses situations : établir un diagnostic, évaluer la sévérité d'une pathologie, l'efficacité d'un traitement. À l'opposé, l'implantation de techniques de physicochimie comme la diffraction des rayons $\mathrm{X}$ ou la spectroscopie infrarouge à transformée de Fourier au sein de l'hôpital reste une exception. Deux spécialités médicales sont en pointe dans ce domaine. Dans le cas de greffes de foie réalisées au centre hépatobiliaire de l'hôpital Paul-Brousse, à Villejuif, l'évaluation de la qualité du greffon est réalisée en routine hospitalière par le biais de la spectroscopie infrarouge et non pas à l'aide d'un microscope optique après coloration des tissus [18]. L'autre application majeure est liée à l'analyse morpho-constitutionnelle qui s'affirme comme l'une des plus belles réussites d'insertion de la physicochimie à l'hôpital. En effet, c'est par dizaine de milliers que se comptent les patients souffrant de lithiase qui ont pu bénéficier de ces travaux. De nos jours, deux spectromètres infrarouge dont l'un de dernière génération sont implantés à l'hôpital Tenon dans le service des explorations fonctionnelles multidisciplinaires du Pr. J.P. Haymann. Ces dispositifs sont ouverts à d'autres disciplines.

\subsection{Le clinicien face aux grands instruments.}

Sur le plan de la recherche académique, plusieurs praticiens hospitaliers participent à des expériences sur de grands instruments. Nous avions déjà souligné l'importance du rayonnement synchrotron et du réacteur de recherche Orphée comme sondes privilégiées de la matière [19]. Cette implication des cliniciens s'accompagne d'échanges fructueux effectués avec les scientifiques responsables des lignes de lumière (ou de neutrons) durant les semaines d'expériences. À ce propos, la première application du CNRSS a porté sur la caractérisation d'une plaque de Randall(PR), l'un des problèmes majeurs en pathologie lithiasique [20].

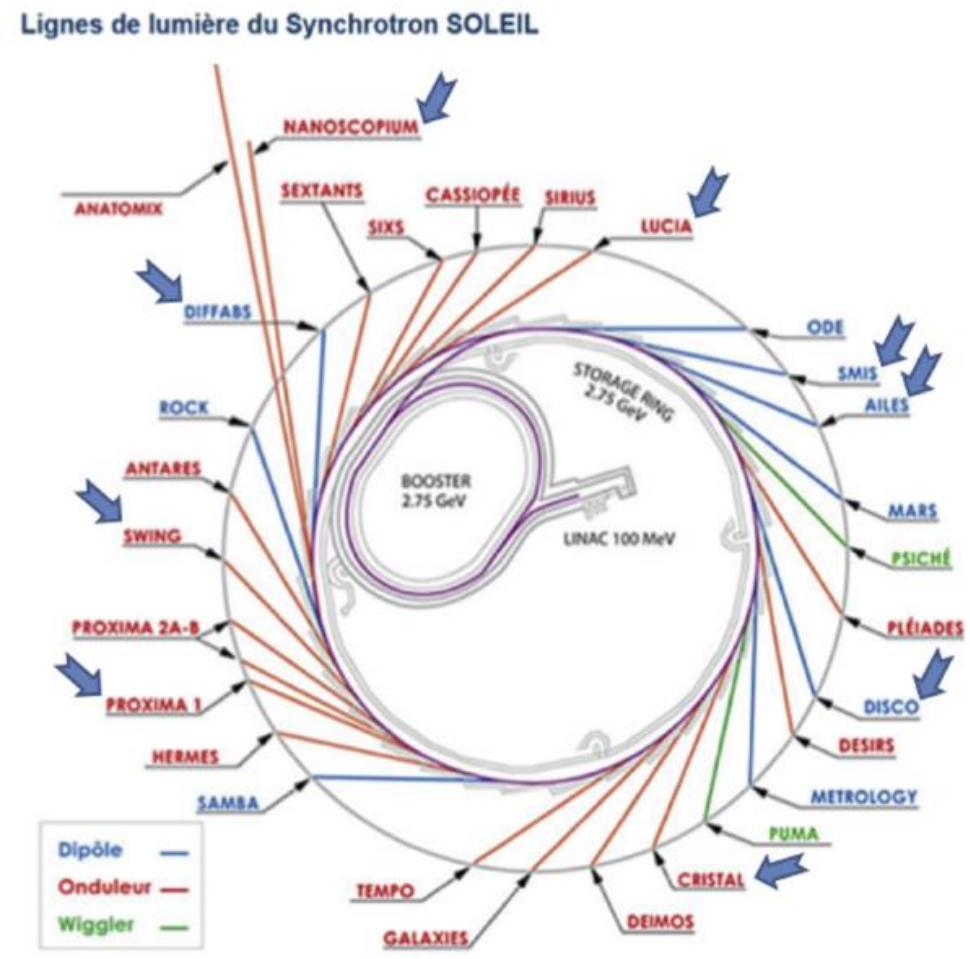

Figure 1. Lignes de lumière du CNRSS qui ont fait l'objet d'une demande de faisceau sont indiquées par la flèche bleue. 
Afin de donner au lecteur un aperçu des techniques mises en œuvre (Fig. 1), citons quelques problématiques médicales [21] :

- la PR par $\mu$ Spectroscopie d'absorption X ( $\mu$ SAX) au seuil K du Ca [21] ;

- la présence de dépôts anormaux dans les biopsies rénales par $\mu$ IRTF [22] ;

- la présence d'oxalate dans les biopsies rénales par nanospectroscopie UV visible ;

-la localisation de dépôts d'urate de sodium ou d'ammonium et d'acide urique dans le rein par $\mu$ IRTF[23,24] ;

-la localisation du $\mathrm{Sr}$ dans les apatites pathologiques et physiologiques par $\mu \operatorname{SAX}[25,26]$;

-le rôle du zinc dans les calcifications articulaires par $\mu \mathrm{SAX}$ au seuil $\mathrm{K}$ du Zn [27,28] ;

-le caractère inflammatoire d'anticancéreux à base de Pt par $\mu \mathrm{SAX}$ au seuil $\mathrm{L}_{\mathrm{III}} \mathrm{du} \mathrm{Pt}$ $[29,30]$;

-la présence d'aluminium dans les calculs par $\mu$ fluorescence $X$;

- la caractérisation par $\mu$ IRTF des nodules induits respectivement par le diabète et le myélome dans le développement de la glomérulosclérose ;

-la détermination de structure de nouveaux composés présents dans les calculs [31,32].

D'autres lignes de lumière ont attiré notre attention. Parmi elles, citons AILES (pour parfaire la connaissance structurale des oxalates de calcium), CRISTAL (pour déterminer la structure de nouvelles phases chimiques), DISCO (pour souligner la présence d'oxalate dans les tissus) et plus récemment PROXIMA (pour déterminer la composition chimique de microcristaux présents dans les urines) et NANOSCOPIUM (pour décrire en 3D les calcifications pathologiques). Des expériences ont aussi été conduites à l'ESRF, le centre de rayonnement synchrotron européen. Ces nouvelles opportunités permettent de caractériser toujours plus précisément des objets de plus en plus petits avec désormais la possibilité d'acquérir la distribution et/ou la morphologie de ces objets biologiques en 3 dimensions. Toutes ces données sont discutées au sein même de l'hôpital avec des cliniciens. C'est de la confrontation entre les données physicochimiques et celles issues du dossier médical du patient que s'établissent de nouvelles méthodes de diagnostic [33]. De même, nous avons mis en œuvre la diffraction de neutrons pour apporter au clinicien des éléments de réponse dans le cas des problématiques suivantes :

- la description de spécificités structurales pour les calculs de whewellite liés à différentes formes d'hyperoxalurie dont l'hyperoxalurie primaire [10,34] ;

- la nature des modifications structurales induites par différents médicaments dans le cas de la cystine [35] ;

- l'influence de la transition entre obésité et diabète de type 2 sur les paramètres structuraux de calculs d'acide urique [36].

\subsection{Prendre en compte la complexité des entités pour intéresser le clinicien}

Des échantillons biologiques humains vont être considérés principalement. Cette approche met le patient au centre de cette recherche. Les modèles animaux sont bien sûr partie prenante de cette recherche. Il nous faut donc aborder la complexité de ces biomatériaux. Ceux-ci intègrent le plus souvent plusieurs phases organiques et inorganiques avec une structure hiérarchique allant du nanomètre au macroscopique. Des molécules métallo-organiques sont également présentes. Tout un jeu de techniques de caractérisation, qu'elles soient classiques i.e. présentes dans un laboratoire ou spécifiques à un grand instrument est donc mis en œuvre. Cette caractérisation multitechniques et multi-échelles nous permet de dépeindre avec finesse les différentes singularités de la calcification (Fig. 2). Nous voudrions maintenant décrire plus en détails quelques résultats afin d'illustrer notre propos. 


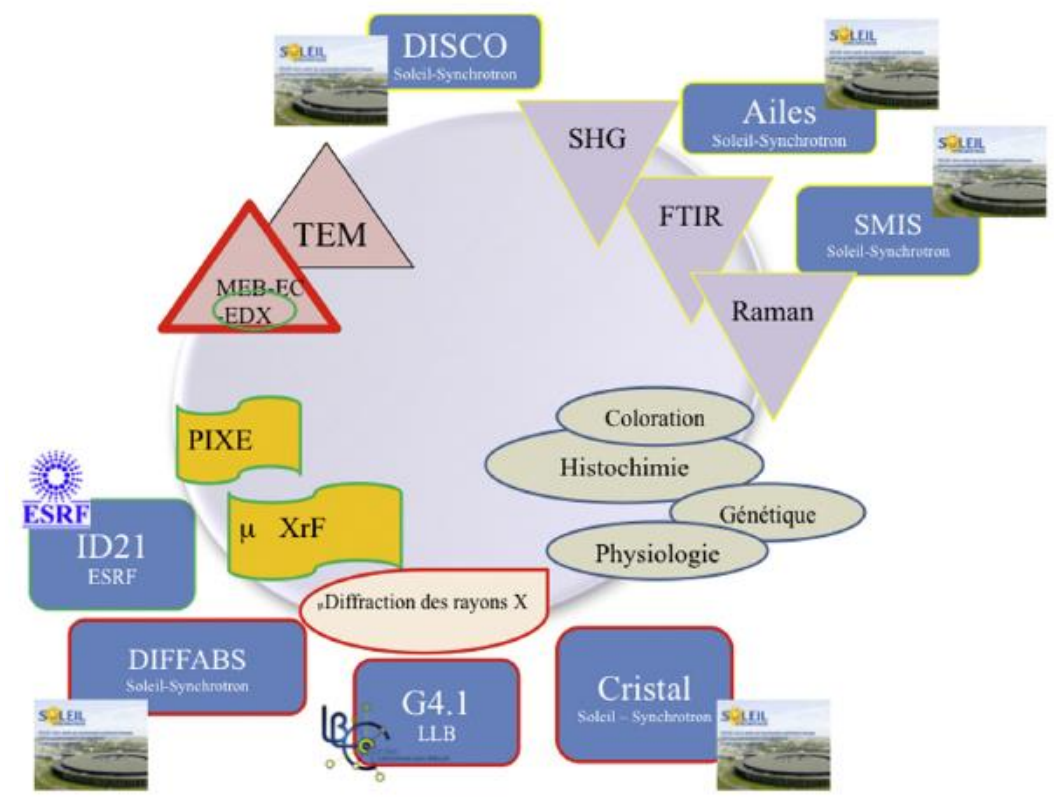

Figure 2. Les calcifications sont caractérisées par les techniques implantées à l'hôpital et par des outils de physicochimie classiques ou implantées sur un grand instrument.

\section{Résultats}

\subsection{Des éléments traces différents pour les calculs de whewellite et de weddellite, signe d'une inflammation tissulaire}

Une abondante littérature est consacrée au rôle d'éléments traces dans la pathogenèse des calcifications [37,38]. Le premier travail (Fig. 3) que nous avons entrepris [39] s'est attaché à déterminer la nature des éléments traces pour les grandes familles définies dans la classification des calculs de Michel Daudon, i.e. les oxalates de calcium (whewellite - calculs de type I et weddellite - type II), les acides uriques et urates (type III), les phosphates (type IV) et la cystine (type V) [6,35].

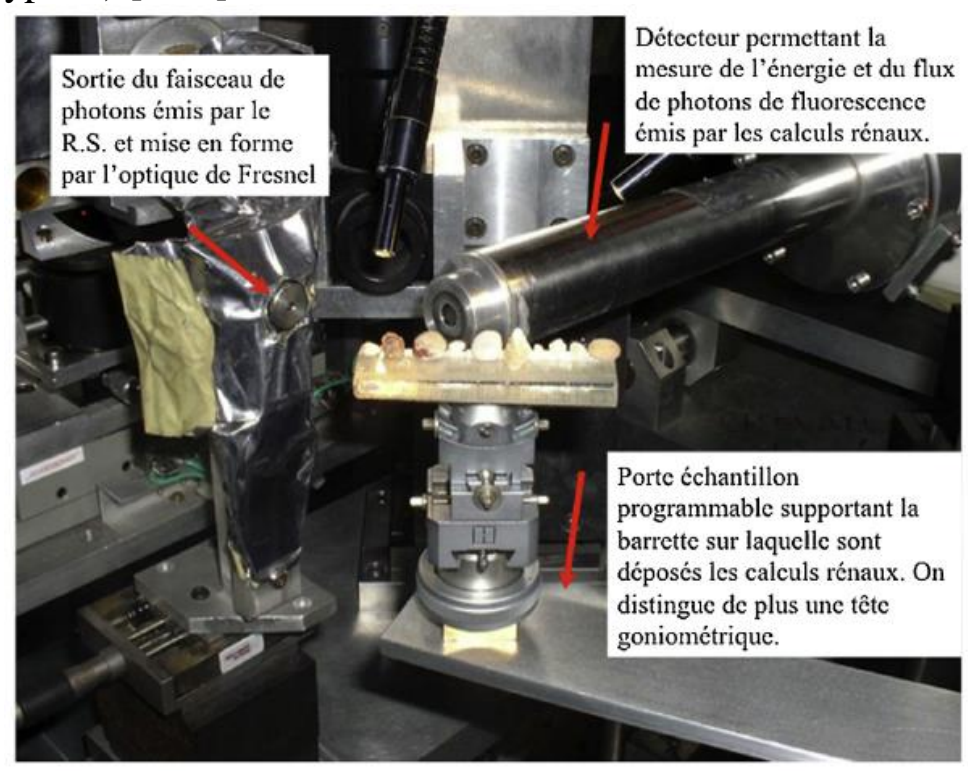

Figure 3. Montage utilisé lors de l'étude des éléments traces sur la pathogenèse des calculs rénaux. On distingue les calculs rénaux placés sur la barrette qui va se déplacer devant le faisceau monochromatique extrait du RS. 
L'analyse fine de résultats récents acquis au laboratoire de physique des solides (LPS) à Orsay sur un prototype (Figure 4) a dévoilé certaines particularités [40].

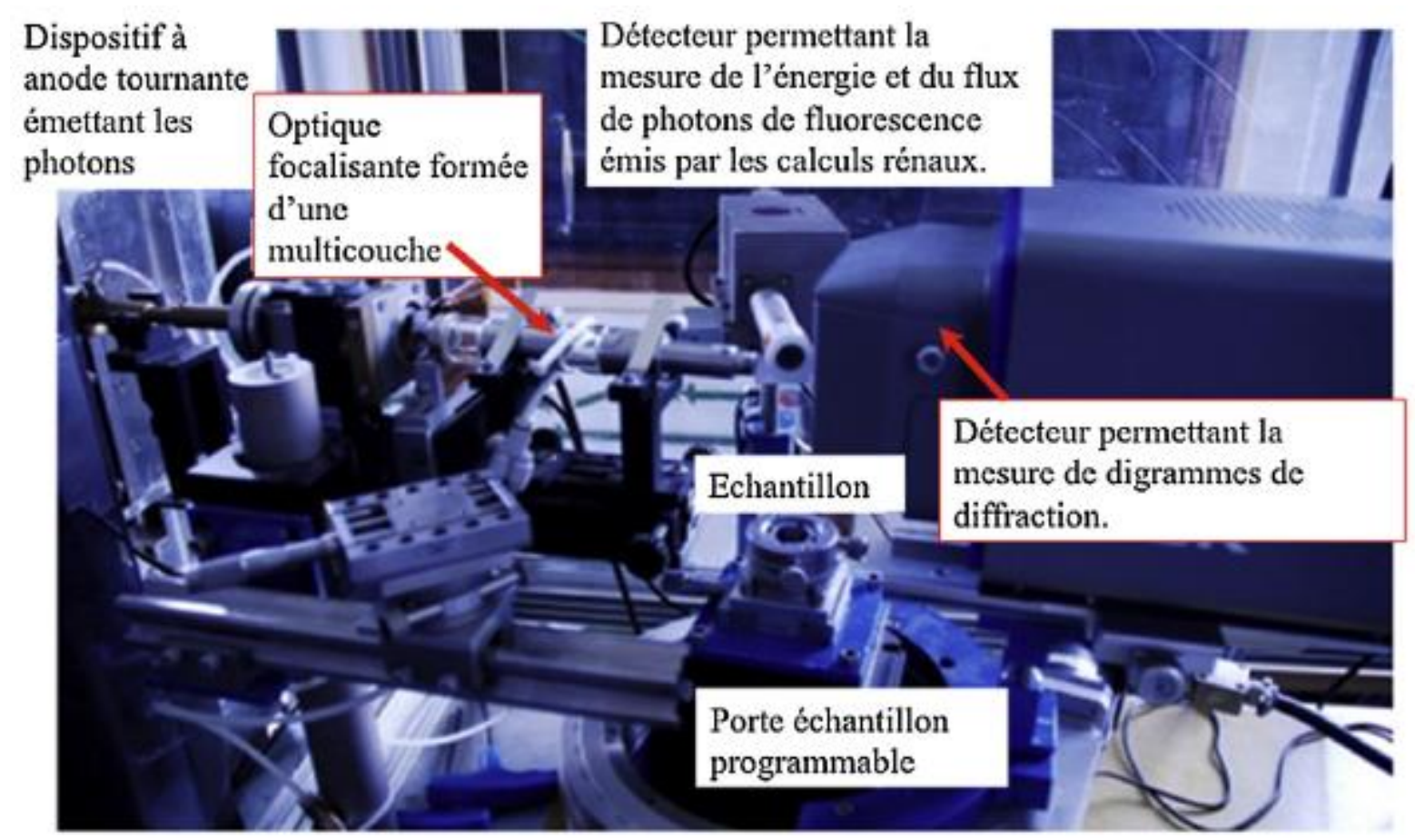

Figure 4. Prototype mis au point au LPS permettant l'acquisition de données de $\mu$ fluorescence $\mathrm{X}$ et $\mu$ diffraction des rayons $\mathrm{X}$.

Ainsi, si l'on compare les teneurs en $\mathrm{Zn}$ des deux composés d'oxalate de calcium, i.e. whewellite et weddellite, on mesure des teneurs en $\mathrm{Zn}$ plus élevées pour la weddellite. Plus précisément, le rapport des aires associées à l'émission de fluorescence $\mathrm{Ca} / \mathrm{Zn}$ est égal à 16,1 $\pm 43,8$ pour la weddellite et à $29,3 \pm 4,9$ pour la whewellite $(\mathrm{p}=0,000053$ pour $\mathrm{Ca} / \mathrm{Zn})$. Cette différence significative suggère des environnements biologiques très différents pour ces deux oxalates. Cette disparité était attendue dans la mesure où une teneur élevée en $\mathrm{Zn}$ signe un contexte d'inflammation. Justement les calculs de weddellite présentent des bipyramides aux angles et arêtes vifs susceptibles d'induire, plus que les calculs de whewellite, une inflammation tissulaire.

\subsection{Plaques de Randall}

Les PR sont des calcifications de l'interstitium de la médullaire profonde décrites pour la première fois par l'urologue Alexander Randall. Elles sont essentiellement composées de carbapatite, même si d'autres composants sont parfois identifiés tels que le phosphate amorphe de calcium carbonaté ou l'urate acide de sodium monohydraté. Leur affleurement à la surface de l'épithélium papillaire les met en contact avec l'urine sursaturée en oxalate de calcium, conduisant à la fixation de cristaux oxalocalciques, essentiellement de whewellite, à la surface de la plaque (Fig. 5). La PR est associée aujourd'hui au principal mécanisme de lithogenèse calcique dans les pays industrialisés [41,42].

Malgré les progrès réalisés dans la compréhension de la composition et de la structure de ces plaques, les mécanismes biochimiques qui sont impliqués dans leur formation restent encore mal connus. L'analyse morpho-constitutionnelle de plus de 30000 calculs montre le caractère épidémique de ce mode de lithogenèse qui affecte aujourd'hui plus de $60 \%$ des sujets lithiasiques jeunes [43-45]. De plus, comme le suggère la teneur élevée en zinc de la 
carbapatite qui constitue la $\mathrm{PR}$, la présence de ces dépôts interstitiels génère vraisemblablement une réaction inflammatoire locale [46]. Sur la Fig. 5A, on aperçoit une PR localisée au fond d'une dépression en surface d'un calcul. Sa structure à l'échelle mésoscopique résulte en fait d'une agglomération de tubules calcifiés soit pleins soit vides (Fig. 5E). Cette image illustre l'impact de la PR située dans l'interstitium sur l'environnement et le fonctionnement des néphrons.

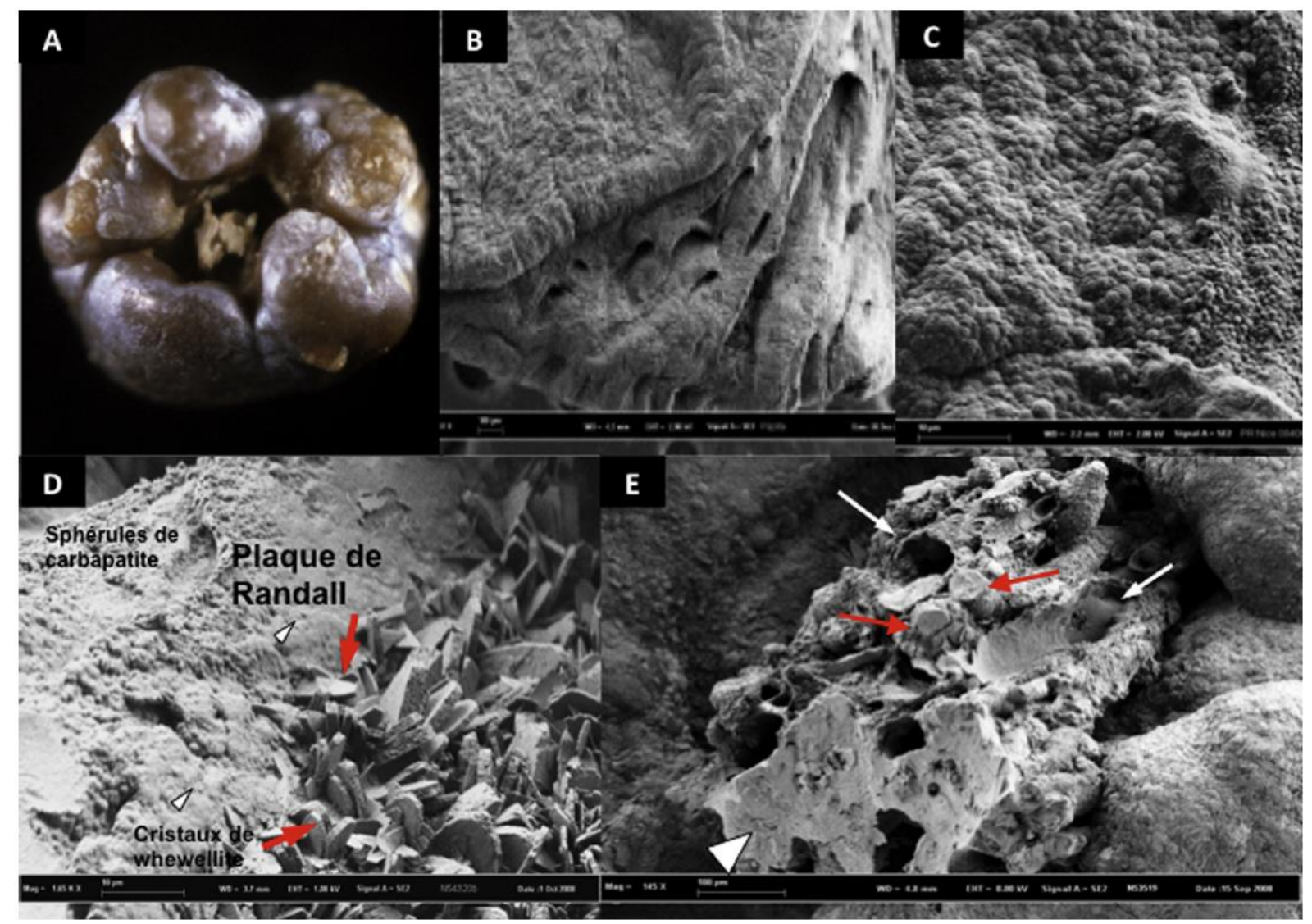

Figure 5. A. Calcul de whewellite avec une PR dans la dépression superficielle (ombilication papillaire). B. Fragment de papille saine vu au microscope électronique à balayage (MEB) et montrant la sortie des tubes collecteurs par où sort l'urine des néphrons à la surface de la papille. C. Fragment de papille recouvert par un tapis de sphères agglomérées de carbapatite correspondant à une PR ayant affleuré à la surface de l'épithélium papillaire. D. Premiers stades de formation d'un calcul oxalocalcique par agglomération de cristaux de whewellite qui viennent s'enficher dans la plaque superficielle de carbapatite. E. Calcul de whewellite formé sur une PR où l'on peut voir les tubes et les vaisseaux calcifiés et les bouchons de carbapatite remplissant certains tubes collecteurs.

Elle illustre aussi le fait que la PR est la résultante de la confluence de dépôts interstitiels mais aussi de dépôts intratubulaires qui peuvent contribuer à déposer des particules minérales à la surface de la plaque lorsque celle-ci a rompu l'épithélium papillaire. Les dépôts accumulés à l'intérieur des tubes collecteurs finissent par remplir la lumière tubulaire, contribuant aussi à altérer le fonctionnement des néphrons concernés. Comme le montre la Fig. 5D, la formation du calcul débute par une accumulation de cristallites micrométriques d'oxalate de calcium englués dans le mélange organophosphatique que constitue la plaque de Randall parvenue à la surface de l'épithélium et qui recouvre en partie celui-ci (Fig. 5C). La Fig. 5E illustre le fait qu'un calcul qui se détache de la papille peut 
entraîner avec lui plusieurs dizaines de tubes collecteurs, vaisseaux et anses de Henlé calcifiés.

\subsection{Les calcifications ectopiques d'oxalates de calcium présentes dans les biopsies rénales}

En une décennie, nous sommes passés d'une recherche centrée sur les concrétions de dimension macroscopique à une recherche dédiée aux calcifications ectopiques de dimension micrométrique. Le but ultime est toujours de concilier physiologie et physicochimie (et parfois génétique) à des échelles cellulaires afin de résoudre un problème posé parle clinicien. Pour cela, il nous a fallu défaire quelques verrous technologiques liés à la préparation des échantillons afin de préserver leur état physico-chimique tout en les rendant compatibles avec les techniques de caractérisation. Les biopsies rénales ont fait l'objet de toute notre attention et nous présentons quelques résultats ci-après.

La biopsie rénale est une procédure diagnostique essentielle dans la pratique de la néphrologie moderne. Elle permet soit de poser un diagnostic de maladie rénale, soit de suivre son évolution. Dans le cas d'un rein natif, elle est souvent indispensable pour le diagnostic précis d'une néphropathie, glomérulaire ou tubulo-interstitielle, ou pour évaluer l'atteinte rénale afin d'adapter la thérapeutique. Dans le cas d'un rein greffé, elle est largement utilisée pour le diagnostic de rejet ou pour guider les traitements immunosuppresseurs [47]. Lors de cet acte médical, le clinicien prélève un petit morceau de rein $( \pm 15 \mathrm{~mm}$ de long sur 2-3 $\mathrm{mm}$ de large) pour l'analyser au microscope optique. À partir de cet échantillon, il est aisé d'extraire une coupe de $5 \mu \mathrm{m}$ d'épaisseur pour effectuer toute une série de caractérisations. Notons que certaines mesures comme celles basées sur la fluorescence $\mathrm{X}$ sont non destructives et peuvent s'effectuer directement sur le bloc de paraffine contenant la biopsie qui sera ainsi rendu intact au service d'anatomopathologie.

Entre 2009 et 2011, les mesures effectuées sur la ligne infrarouge SMIS du CNRSS [13] ont concerné 24 biopsies rénales, l'analyse des données a montré une grande diversité chimique des calcifications ectopiques rénales. Parmi les nouvelles phases chimiques identifiées, citons la silice amorphe, l'urate de sodium monohydraté, l'acide methyl-1 urique et trois composés calciques, i.e. la whitlockite, le phosphate octocalcique pentahydraté et le phosphate amorphe de calcium carbonaté (PACC). Dans un second temps, la présence d'un microspectromètre infrarouge à transformée de Fourier installé à l'hôpital a permis d'accroître considérablement le nombre de biopsies étudiées, plus de 800 à ce jour. Parmi elles, 232 biopsies rénales ont été analysées. Cette nouvelle étude a souligné la diversité considérable des espèces cristallines mises en évidence dans le tissu rénal [48]. Vingt-deux corps ont été ainsi identifiés. Cette diversité permet de comprendre la difficulté de poser un diagnostic par des colorations histologiques qui ne permettent pas de caractériser de manière pertinente les phases minérales ou organiques présentes dans les tissus.

Cette diversité chimique reflète souvent des environnements pathologiques différents, et donc importants à reconnaître pour ce qu'ils sont. Elle rend le travail de l'anatomopathologiste difficile. Les méthodes de coloration sont donc inappropriées car seule une identification précise des phases cristallines mène à la pose d'un diagnostic médical juste. Dans le service des explorations fonctionnelles de l'hôpital Tenon, untel appareil est accessible. Ainsi, ces analyses montrent que certaines espèces sont associées à des contextes pathologiques très particuliers comme cela a été discuté récemment [48]. En complément de ces mesures infrarouge, nous avons procédé, pour une série limitée de biopsies de patients associées à des hyperoxaluries, à des observations par MEB.

Cette double approche permet d'identifier les composés chimiques présents dans les dépôts anormaux et d'en décrire la morphologie à des échelles subcellulaires. Les mêmes biopsies ont étudiées par spectroscopie infra-rouge et microscopie électronique à balayage (Fig. 6). 


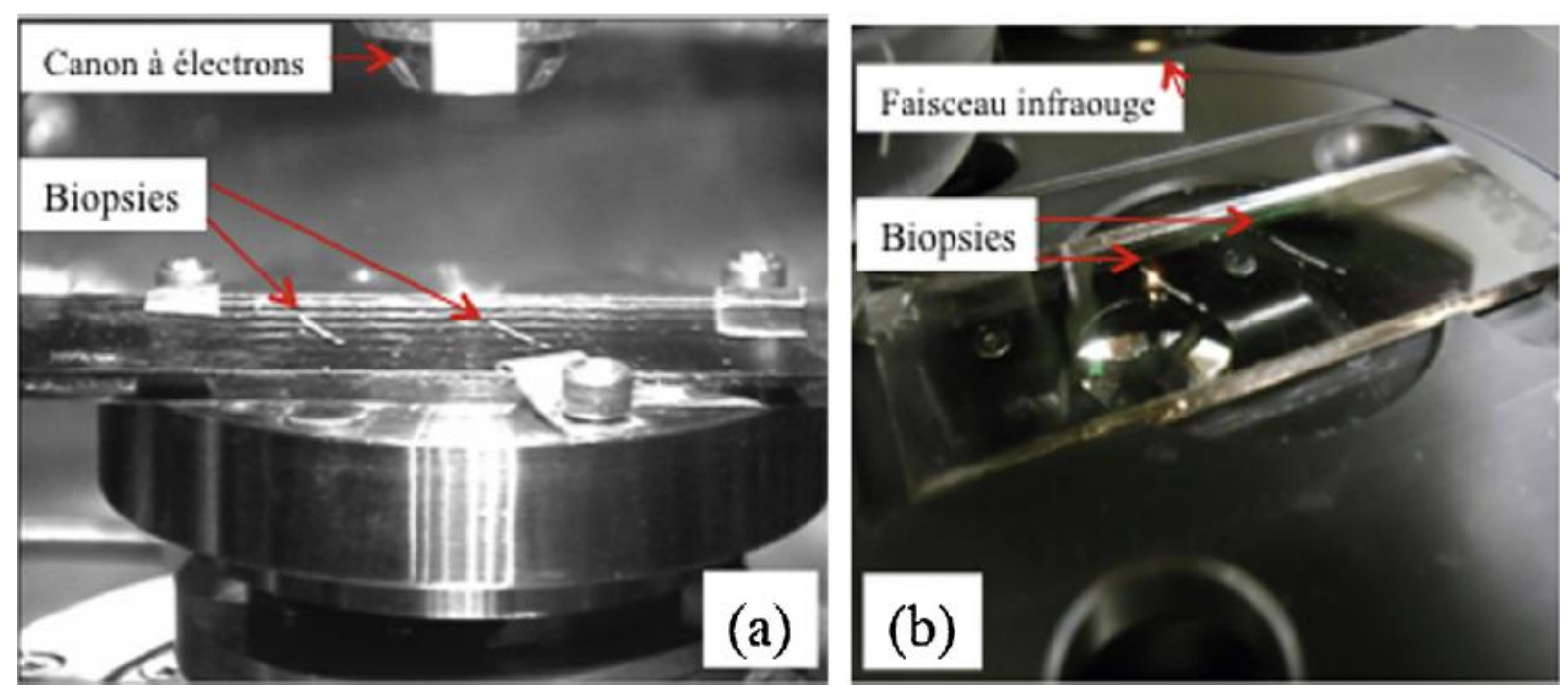

Figure 6. a : biopsies placées dans l'enceinte sous vide du MEB du LPS ; b : biopsies placées sur le support du spectromètre infrarouge implanté sur le CNRSS sur le plateau d'Orsay.

Dans la mesure où le microscope dispose d'un détecteur de fluorescence $X$, il est possible de collecter des informations relatives à la répartition spatiale des éléments. Si une expérience de fluorescence $\mathrm{X}$ induite par des photons est plus sensible qu'une expérience de fluorescence induite par des électrons, cette dernière permet de collecter des informations avec une plus grande résolution spatiale.

Pour savoir si la morphologie des dépôts d'oxalate de calcium dépend de son étiologie, nous considérons des patients présentant des hyperoxaluries associées à différentes pathologies (Tableau 1).

Tableau 1. Etiologie du jeu de biopsies sélectionnées pour cette étude

\begin{tabular}{|ll|}
\hline Biopsie & Etiologie \\
B274 & Consommation excessive de rhubarbe \\
B279 & Hyperoxalurie non étiquetée \\
B292 & Oxalose éthylène glycol \\
B305, B317 & Néphrocalcinose post transplantation rénale \\
B329 & Tunisie - hyperoxalurie primaire \\
B364 & Mucoviscidose \\
B379 & Chirurgie bariatrique \\
B380 & Cancer gastrique \\
B386 & Néphrocalcinose post transplantation rénale \\
\hline
\end{tabular}

Certaines biopsies présentent des dépôts de natures chimiques différentes. C'est le cas de la biopsie B317 (Fig. 7). Des dépôts d'oxalate de calcium repérés grâce à la raie d'absorption positionnée à $780 \mathrm{~cm}^{-1}$ (Fig. 7A et B) et de phosphate de calcium (Fig. $7 \mathrm{C}$ et D) sont identifiés. 

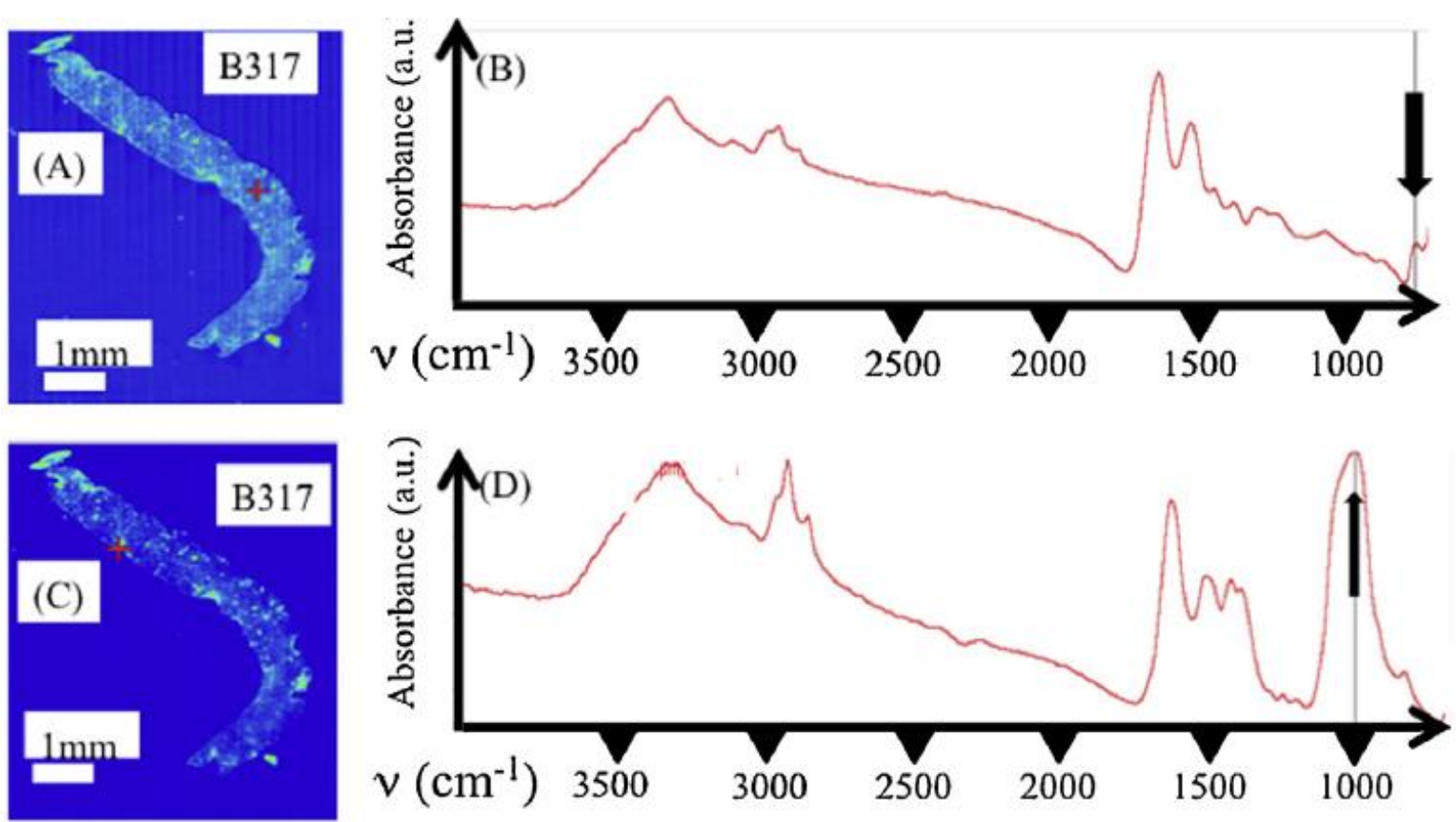

Figure 7. B317 : la spectroscopie IR met en évidence la présence de dépôts anormaux constitués, soit d'oxalate de calcium monohydraté (A et B), soit de phosphates de calcium carbonatés, carbapatite et PACC (C et D).

Sur la biopsie B292 (Fig. 8A), on observe une calcification de l'assise tubulaire d'un tube (Fig. 8B) ainsi qu'une calcification remplissant toute la lumière du tube. Les morphologies associées semblent très différentes (Fig. 8, Cet D).

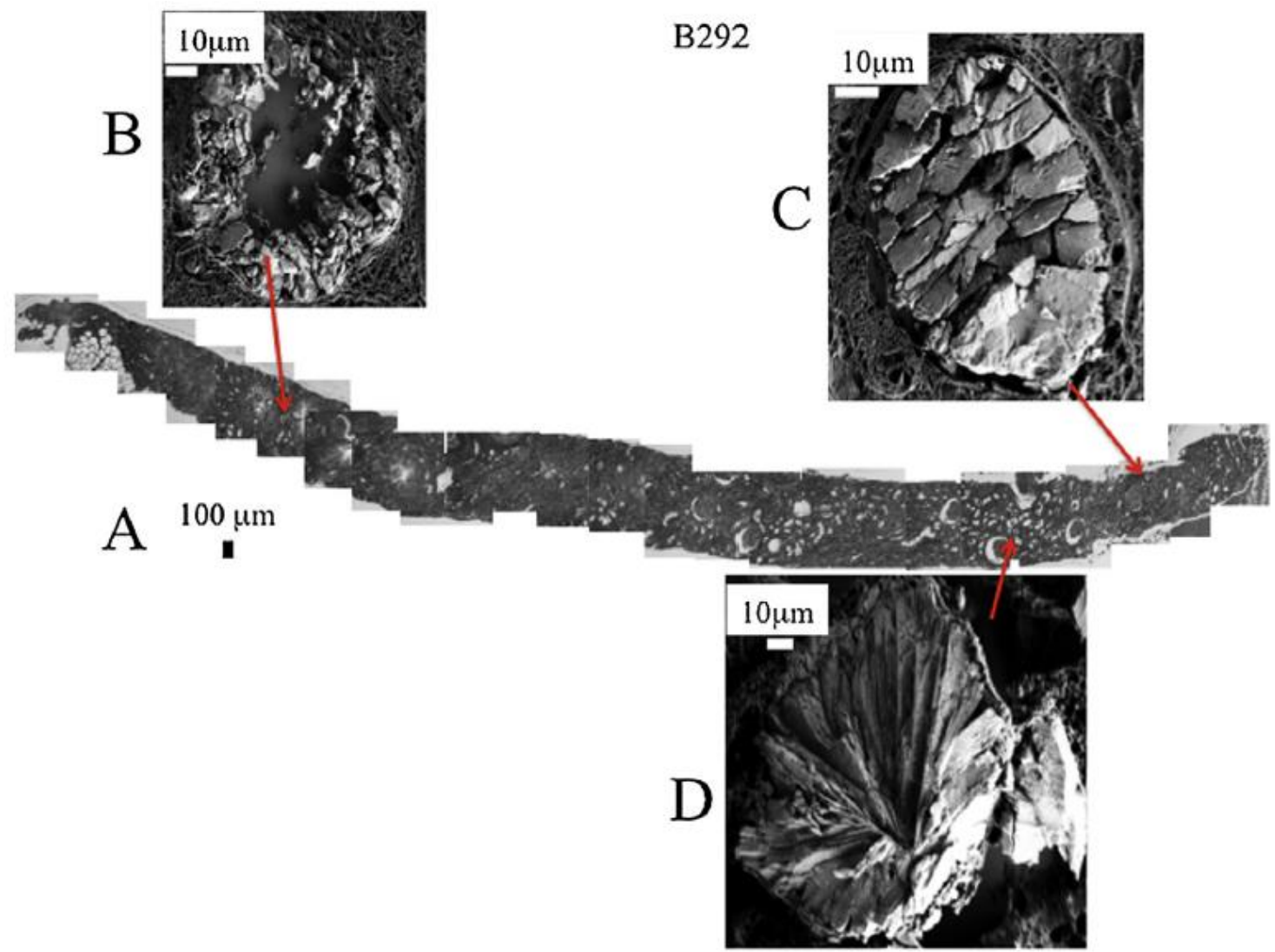

Figure 8. Visualisation par MEB de dépôts de différentes morphologies. 
Sur le plan physicochimique, il existe des différences notables entre l'environnement biochimique d'une concrétion et celui d'une calcification ectopique. Lorsque l'on considère l'environnement biochimique d'une concrétion, les équations qui régissent la sursaturation peuvent simplement s'appliquer pour rendre compte de sa genèse et l'on peut en modulant certains paramètres comme l'hydratation du patient ou le $\mathrm{pH}$ de son urine restreindre ou inhiber complètement la formation de la concrétion. Dans une certaine mesure, on peut considérer que la concrétion se forme dans un volume fini de solution (l'un des calices, le bassinet ou la vessie). La pathologie va modifier certains paramètres biochimiques de l'ensemble de cette solution.

Dans le cas de la calcification ectopique qui va se former au sein du tissu rénal et donc généralement dans le néphron, la situation est complètement différente. Le long de l'unité fonctionnelle, l'environnement biochimique est modulé de manière significative par plusieurs processus d'absorption et de sécrétion. Au sein d'un glomérule, un processus de filtration unidirectionnel passif et non sélectif sous l'effet de la pression glomérulaire s'effectue. Au niveau du tube contourné proximal, de nombreuses substances vont être réabsorbées parmi lesquelles $\mathrm{Na}^{+}, \mathrm{K}^{+}, \mathrm{H}_{2} \mathrm{O}$, bicarbonates et $\mathrm{Cl}^{-}$, glucose, acides aminés, vitamines. . . En ce qui concerne l'anse de Henlé, qui fait suite au tube proximal on observe une réabsorption de l'eau pour la branche descendante et une réabsorption de $\mathrm{Na}^{+}, \mathrm{K}^{+}, \mathrm{Cl}^{-}$pour la branche ascendante. Finalement, les protons ne seront réabsorbés qu'au niveau du tube collecteur distal.

Tous ces échanges, auxquels s'ajoutent aussi des sécrétions sélectives de certains ions entraînent des modifications de la composition du flux intratubulaire et, potentiellement, de la sursaturation en différentes espèces cristallines, sans compter les interactions entre germes cristallins éventuellement formés et les macromolécules protéiques ou glucidiques présentes à la surface des cellules et dans le fluide tubulaire. Les altérations fonctionnelles ou structurelles rénales peuvent aussi être induites par des molécules d'origine exogène (médicaments ou leurs métabolites). Dans ce cas, les lésions associées (réversibles ou définitives) touchent l'ensemble du néphron (glomérule, tubule) ainsi que l'interstitium. Récemment, nous nous sommes intéressés aux dépôts associés à ces néphrotoxicités d'origine médicamenteuse, Atazanavir et Foscarnet (Fig. 9) principalement. À la demande des cliniciens, nous allons continuer cet axe de recherche.

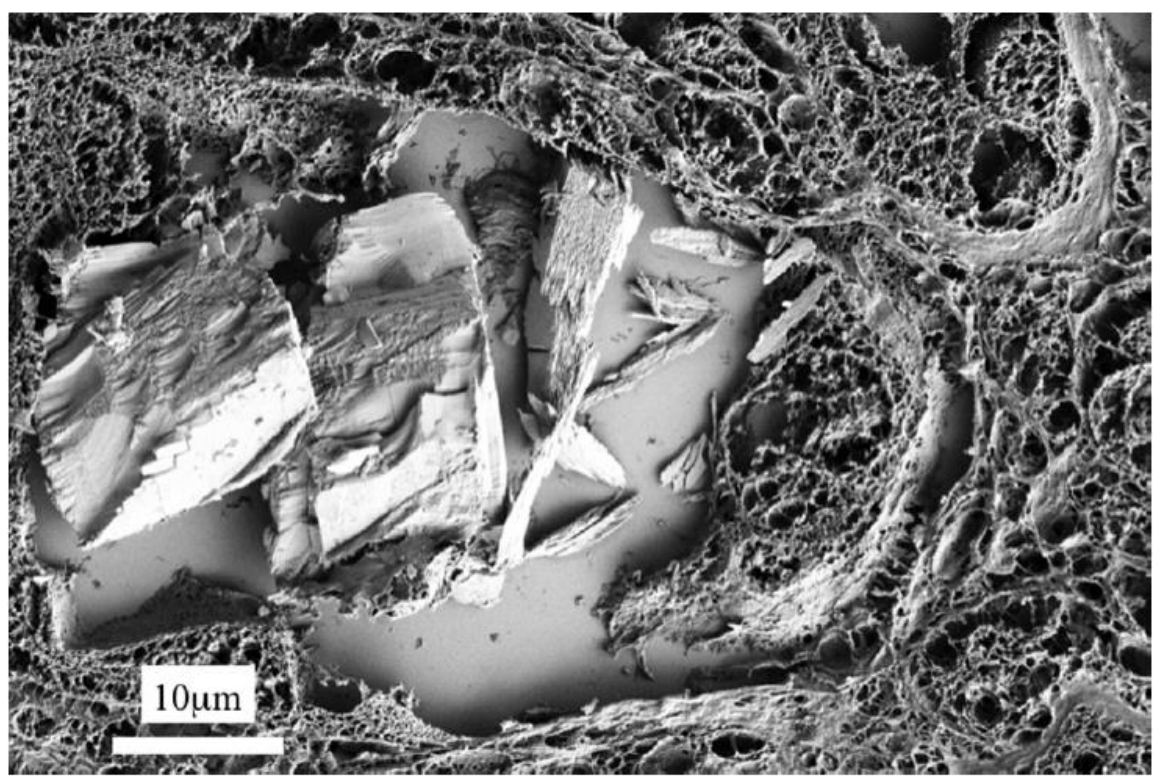

Figure 9. Topologie de dépôts de Foscarnet par MEB. 


\section{Conclusion}

Au-delà des progrès cognitifs et des applications médicales à moyen terme, l'ensemble des travaux mentionnés ci-dessus a des implications immédiates pour la prise en charge du patient. À titre d'exemple, la première biopsie rénale analysée par l'imageur infrarouge de l'hôpital Tenon a permis d'identifier des cristaux de dihydroxyadénine présents dans le transplant rénal d'un enfant greffé en raison des conséquences d'une autre maladie génétique. L'analyse infrarouge a ainsi permis de porter le diagnostic de mutation du gène codant pour l'adénine phosphoribosyltransférase.

Cette pathologie du métabolisme des purines savait probablement contribué à la destruction des reins natifs de l'enfant et menaçait de détruire le greffon rénal. Les techniques conventionnelles d'anatomopathologie ne peuvent établir ce diagnostic. Cette pathologie est curable par l'allopurinol. Ce traitement a été donné très rapidement à l'enfant et lui a permis ainsi de récupérer une fonction rénale pratiquement normale. Ces travaux démarrés il y a 10 ans $[49,50]$ sur la PR[21,42-46] ont permis de concevoir un nouveau paradigme. Nos prochaines recherches tendent vers une description 3Ddes calcifications à l'échelle nanométrique. En cela, la plateforme PIPA basée sur de la tomographie X sur animal vivant dirigée par le Pr. C. Chaussain constitue une opportunité sans équivalent. Une première moisson de résultats est associée aux travaux conduits par le Pr. E. Letavernier sur la plaque de Randall. Avec M. Refregiers, scientifique responsable de la ligne DISCO du CNRSS, les premières reconstructions $3 \mathrm{D}$ à partir de l'émission UV ont été réalisées.

C'est dans cette perspective que s'inscrivent les tests sur la ligne de lumière NANOSCOPIUM avec A. Somogy et K. Medjoubi, scientifiques de la ligne. Cette dernière demande s'inscrit dans le cadre de la thèse du Dr. E. Estève sur une nouvelle génération de vecteurs thérapeutiques dans le service du Pr. P. Ronco à qui cette recherche sur les calcifications pathologiques doit beaucoup.

\section{Remerciements}

Cette recherche a été soutenue par trois ANR (ANR-09-BLAN-0120-02, ANR-12BS08-0022-03, ANR-13-JSV1-0010-01), un projet CORDDIM (2013-COD13004) et un projet Convergence de l'université Pierre-et-Marie-Curie, la Société de Néphrologie (Genzyme grant), et l'Académie nationale de médecine (Nestlé-Waters award).

\section{Déclaration de liens d'intérêts}

Les auteurs déclarent ne pas avoir de liens d'intérêts. 


\section{Références}

[1] J. Friedel, Communications privées.

[2] http://www.pasteur.fr/fr.

[3] http://www.inserm.fr/thematiques/technologies-pour-la-sante/dossiers-dinformation/imagerie-fonctionnelle-biomedicale.

[4] http://www.annales.org/archives/x/cfriedel.html.

[5] Daudon M, Traxer O, Jungers P. Lithiase urinaire. 2eed. Paris:Lavoisier, Médecine Sciences; 2012 [672 p.].

[6] Daudon M, Bader CA, Jungers P. Urinary calculi: review of classification methods and correlations with etiology. Scanning Microsc 1993;7:1081-104.

[7] Ceballos-Picot I, Perignon JL, Hamet M, Daudon M, Kamoun P.2,8-Dihydroxyadenine urolithiasis, an underdiagnosed disease. Lancet 1992;339:1050-1.

[8] Daudon M, Estépa L, Viard JP, Joly D, Jungers P. Urinary stones in HIV-1-positive patients treated with indinavir. Lancet1997;349:1294-5.

[9] Daudon M, Reveillaud R, Jungers P. Piridoxilate-associated calcium oxalate urinary calculi: a new metabolic drug-induced nephrolithiasis. Lancet 1985;325:1338-9.

[10] Daudon M, Jungers P, Bazin D. Peculiar morphology of stones in primary hyperoxaluria. New Engl J Med 2008;359:100-2.

[11] http://www.synchrotron-soleil.fr/.

[12] http://www-llb.cea.fr/.

[13] Dessombz A, Méria P, Bazin D, Daudon M. Prostatic stones: evidence of a specific chemistry related to infection and presence of bacterial imprints. PLoS One 2012;7:e51691.

[14] Dessombz A, Méria P, Bazin D, et al. Diversité chimique des calculs prostatiques : une investigation par MEB et spectroscopie infrarouge. Prog Urol 2011;21:940-5.

[15] Ben Lakhdar A, Daudon M, Mathieu M-C, et al. Underlining the complexity of the structural and chemical characteristics of ectopic calcifications in breast tissues through FESEM and $\mu$ FTIR spectroscopy. C R Chim 2016, http://dx.doi.org/10.1016/j.crci.2015.03.011 [sous presse].

[16] Cros J, Bazin D, Kellum A, Rebours V, Daudon M. Investigation at the micrometer scale of pancreatic calcifications in chronic pancreatitis by ( $\mu$ FTIR spectroscopy and field emission scanning electron microscopy. C R Chim 2016, http://dx.doi.org/10.1016/j.crci.2015.06.015 [sous presse].

[17] Mathonnet M, Dessombz A, Bazin D, et al. Chemical diversity of calcifications in thyroid and hypothetical link to disease. C R Chim 2016, http://dx.doi.org/10.1016/j.crci.2015.02.008 [sous presse].

[18] Debois D, Bralet M-P, Le Naour F, Brunelle A, Laprévote O. In situ lipidomic analysis of nonalcoholic fatty liver by cluster TOF-SIMS imaging. Anal Chem 2009;81:2823-31.

[19] Bazin D, Daudon M, Chevallier P, et al. Les techniques de rayonnement synchrotron au service de la caractérisation d'objets biologiques : un exemple d'application, les calculs rénaux. Ann Biol Clin 2006;64:125-39.

[20] Hasnain S. Current events. J Synchrotron Rad 2007;14:297-8.

[21] Carpentier X, Bazin D, Jungers P, Reguer S, Thiaudière D, Daudon M. The pathogenesis of Randall's plaque: a papillacartography of $\mathrm{Ca}$ compounds through an ex vivo investigation based on XANES spectroscopy. J Synchrotron Rad. 2010;17:374-9.

[22] Dessombz A, Bazin D, Dumas P, Sandt C, Sule-Suso J, Daudon M. Shedding light on the chemical diversity of ectopic calcifications in kidney tissues: diagnostic and research aspects. PLoSONE 2008;6:e28007-15. 
[23] Preitner F, Laverriere A, Metref S, et al. Urate-induced acute renal failure and chronic inflammation in liver-specific Glut9 knockout mice. Am J Physiol Renal Physiol2013;305:F786-95.

[24] Bazin D, Letavernier E, Jouanneau Ch, et al. New insights on the presence of sodium hydrogen urate monohydrate inRandall's plaque. C R Chim 2016, http://dx.doi.org/10.1016/j.crci.2015.02.010.

[25] Bazin D, Daudon M, Chappard Ch, Rehr JJ, Thiaudière D, Reguer S. The status of strontium in biological apatites: an XANES investigation. J Syn. Rad 2011;18:912-8.

[26] Bazin D, Dessombz A, Nguyen C, et al. The status of strontium in biological apatites: an XANES/EXAFS investigation. J. Syn. Rad 2014;21:136-42.

[27] Nguyen C, Ea HK, Thiaudière D, et al. Calcifications in human osteoarthritic articular cartilage: ex vivo assessment of calcium compounds using XANES spectroscopy. J Syn. Rad 2011;18:475-80.

[28] Dessombz A, Nguyen Ch, Ea HK, et al. Combining $\mu$ X-ray fluorescence, $\mu$ XANES and $\mu \mathrm{XRD}$ to shed light on $\mathrm{Zn}^{2+}$ cations in cartilage and meniscus calcifications. $\mathrm{J}$ Trace Elem Med Biol2013;27:326-33.

[29] Esteve E, Bazin D, Jouanneau C, et al. How to assess the role of $\mathrm{Pt}$ and $\mathrm{Zn}$ in the nephrotoxicity of $\mathrm{Pt}$ anti-cancer drugs? An investigation combining $\mu \mathrm{XRF}$ and statistical analysis. Part I: Mice. C R Chim 2016, http://dx.doi.org/10.1016/j.crci.2016.03.014 [sous presse].

[30] Esteve E, Bazin D, Jouanneau C, et al. How to assess the role of $\mathrm{Pt}$ and $\mathrm{Zn}$ in the nephrotoxicity of $\mathrm{Pt}$ anti-cancer drugs? An investigation combining $\mu \mathrm{XRF}$ and statistical analysis. Part II : Clinical application. C R Chim 2016, http://dx.doi.org/10.1016/j.crci.2016.02.015 [sous presse].

[31] Le Bail A, Bazin D, Daudon M, Brochot A, Robbez-Masson V ,Maisonneuve V. Racemic calcium tartrate tetrahydrate [form(II)] in rat urinary stones. Acta Cryst 2009;B65:350-4.

[32] Daudon M, Bazin D, Adil K, Le Bail A. 7,9-Bis(hydroxymethyl)-7H-purine-2,6,8 $(1 \mathrm{H}, 3 \mathrm{H}, 9 \mathrm{H})$ trione. Acta Cryst 2011;E67:o1458.

[33] http://www2.cnrs.fr/presse/communique/2354htm.

[34] Daudon M, Bazin D, André G, et al. Examination of whewellite kidney stones by scanning electron microscopy and powder neutron diffraction technique. J Applied Cryst2009;42:109-15.

[35] Bazin D, Daudon M, André G, Weil R, Véron E, Matzen G. Therapy modifies cystine kidney stones at the macroscopic scale. Do such alterations exist at the mesoscopic and nanometre scale? J Applied Cryst 2014;47:719-25.

[36] Daudon M, Letavernier E, Weil R, Véron E, Matzen G, André G,et al. Type 2 diabetes and uric acid stones: a powder neutron diffraction investigation. C R Chim 2016, http://dx.doi.org/10.1016/j.crci.2015.04.006 [sous presse].

[37] Ramaswamy K, Killilea DW, Kapahi P, Kahn AJ, Chi T, Stoller ML.The elementome of calcium-based urinary stones and its role in urolithiasis. Nat Rev Urol 2015;12:543-57.

[38] Bazin D, Carpentier X, Brocheriou I, et al. Revisiting the localisation of $\mathrm{Zn}^{2+}$ cations sorbed on pathological apatite calcifications made through X-ray absorption spectroscopy. Biochimie 2009;91:1294-300.

[39] Bazin D, Chevallier P, Matzen G, Jungers P, Daudon M. Heavy elements in urinary stone. Urol Res 2007;35:179-84.

[40] Rouzière S, Bazin D, Daudon M. In-lab X-ray fluorescence and diffraction techniques for pathological calcifications. C R Chim2016, http://dx.doi.org/10.1016/j.crci.2015.05.013. 
[41] Evan AP, Evan AP, Lingeman JE, Coe FL, et al. Randall's plaque of patients with nephrolithiasis begins in basement membranes of thin loops of Henle. J Clin Invest 2003;111:607-16.

[42] Daudon M, Traxer O, Jungers P, Bazin D. Stone morphology suggestive of Randall's plaque. AIP Conf Proc 2007; 900:26-32.

[43] Daudon M, Traxer O, Williams JC, Bazin D. Randall's plaques. In: Rao PN, Preminger GM, Kavanagh JP, editors. Urinary tract stone disease. London: Springer; 2011. p. 103-12.

[44] Daudon M, Bazin D, Letavernier E. Randall's plaque as the origin of calcium oxalate kidney stones. Urolithiasis 2015;43:5-10.

[45] Letavernier E, Vandermeersch S, Traxer O, et al. Demographics and characterization of 10,282 Randall plaque-related kidney stones. Medicine 2015;94(10):e566.

[46] Carpentier X, Bazin D, Combes C, et al. High Zn content of Randall's plaque: a $\mu-X-$ ray fluorescence investigation. J Trace Elem Med Biol 2011;25:160-5.

[47] http://www.soc-nephrologie.org/PDF/esociete/groupes/biopsie/info QBR.pdf.

[48] Bazin D, Daudon M. Les pathologies microcristallines et les techniques de physicochimie : quelques avancées. Ann Biol Clin2015;73:517-34.

[49] Bazin D, Daudon M, Combes C, Rey C. Characterization andsome physicochemical aspects of pathological microcalcifications. Chem Rev 2012;112:5092-120.

[50] Bazin D, Daudon M. Pathological calcifications and selected examples at the medicinesolid-state physics interface. J PhysD Appl Phys 2012;45:383001-10. 